\title{
Automatic Detection of Microaneurysms AND HEMORRHAGES IN COLOR EYE FUNDUS IMAGES
}

\author{
Sérgio Bortolin Júnior ${ }^{1}$ and Daniel Welfer ${ }^{2}$ \\ Programa de Pós-Graduação em Engenharia Elétrica (PPGEE), Universidade Federal do \\ Pampa, Brazil \\ ${ }^{1}$ LAPIA - Laboratório de Processamento de Imagem Aplicado \\ ${ }^{2}$ GPSEI - Grupo de Pesquisa em Sistemas Eletrônicos
}

\begin{abstract}
This paper presents an approach for automatic detection of microaneurysms and hemorrhages in fundus images. These lesions are considered the earliest signs of diabetic retinopathy. The diabetic retinopathy is a disease caused by diabetes and is considered as the major cause of blindness in working age population. The proposed method is based on mathematical morphology and consists in removing components of retinal anatomy to reach the lesions. This method consists of five stages: a) pre-processing; $b$ ) enhancement of low intensity structures; c) detection of blood vessels; d) elimination of blood vessels; e) elimination of the fovea. The accuracy of the method was tested on a public database of fundus images, where it achieved satisfactory results, comparable to other methods from the literature, reporting $87.69 \%$ and $92.44 \%$ of mean sensitivity and specificity, respectively.
\end{abstract}

\section{KEYWORDS}

Image Processing, Mathematical Morphology, Fundus Images, Microaneurysms, Hemorrhages.

\section{INTRODUCTION}

The World Health Organization (WHO) estimates that there are currently 347 million people suffering from diabetes and projects that this disease will be the seventh leading cause of death worldwide in 2030 [1]. Over the years, patients with diabetes tend to show abnormalities in the retina, developing a complication called Diabetic Retinopathy (DR). DR is one of the most serious diseases affecting the eye, and it is considered the most common cause of blindness in adults between 20 and 60 years of age [2]. Studies show that people above the age of 30 years that suffer from diabetes for more than 15 years have $78 \%$ chance of also having DR. This rate rises to $97 \%$ for people below the age of 30 years that suffer from diabetes for the same period of time [3]. Thus, DR affects adults in working age, being a disease that is strongly associated with inability to work, causing significant costs to institutions and governments. It is estimated that the U.S. government has an annual cost of 500 million dollars in the treatment of visual impairments originated by diabetes [4].

The earliest signs of DR are characterized by the appearance of red lesions on the wall of the blood vessels of the retina [5], and these red lesions are classified in two ways: microaneurysms and hemorrhages. 
The microaneurysms can cause narrowing and sometimes blockage of blood vessels of the retina, besides weakening of the blood vessels wall [6]. These microaneurysms may rupture, causing hemorrhages [6]. According to an international classification of severity levels of DR [7], the microaneurysms are commonly present in the early stages of this disease and the hemorrhages tend to manifest in the later stages.

Early detection as well as the periodic screening of DR potentially helps in reducing the progression of this disease and in preventing the subsequent loss of visual capability [8]. The detection and screening of RD are performed by analysis of fundus images.

Fundus images are captured through an examination which is usually performed in two ways: fundus photography or fluorescein angiography. Fundus photography is a noninvasive technique for photographing the fundus eye and is commonly used in current methods for detection of microaneurysms and hemorrhages, such as $[2,4,5,9]$. Fluorescein angiography is an invasive technique based on intravenous injection of a fluorescent substance, which produces images with enhanced contrast. However, this technique is not recommended for large scale screening of diabetic populations. In addition to possible side effects, such as nausea and vomiting, a mortality of 1:222.000 associated with the intravenous use of fluorescein prohibits the application of this technique for large-scale screening purposes [9].

In Fig. 1 is shown a fundus image captured in a noninvasive way, containing microaneurysms and hemorrhages.

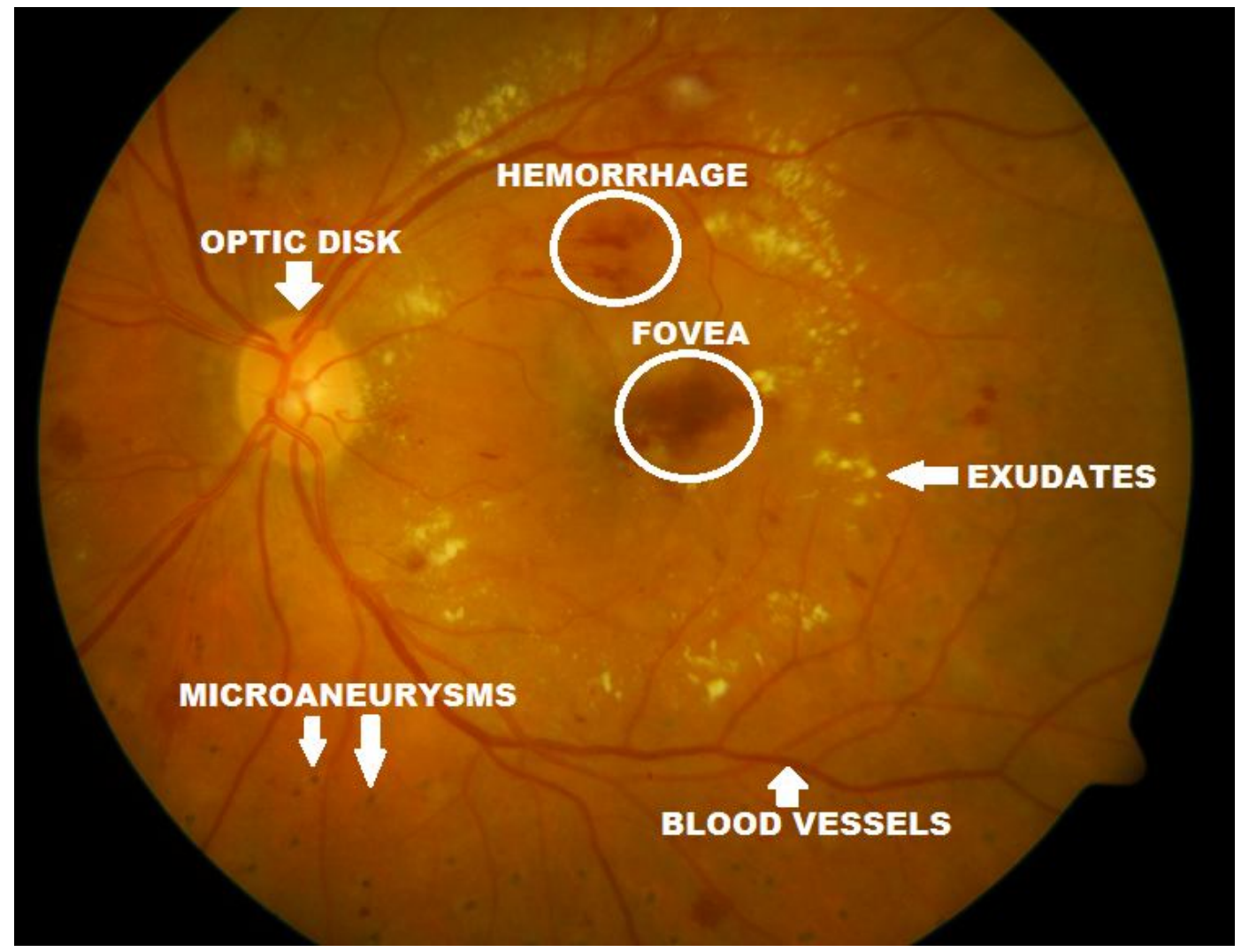

Figure 1. Fundus image containing microaneurysms and hemorrhages 
As shown in Fig. 1, the microaneurysms are small red spots dispersed on the blood vessels wall. On the other hand, the hemorrhages have a larger size, and are characterized by red swellings.

The manual detection of these red lesions is made by ophthalmologists and is a slow and errorprone activity. Some lesions have a very small size and may go unnoticed even by trained specialists. In addition, manual detection may be considered unfeasible in government programs for mass screening of diabetic populations, since it would require a huge amount of doctors and examinations. Thus, it is evident the need of methods for automatic detection of red lesions in fundus images.

This paper is organized as follows: Section 2 presents some current methods for detection of microaneurysms and hemorrhages, emphasizing its main characteristics and common problems in the area; Section 3 explains in details the method for detection of microaneurysms and hemorrhages proposed in this paper; Section 4 shows the results achieved by the proposed method and compares these results with other methods in the literature; and, finally, Section 5 presents the conclusion of this article.

\section{RELATED WORK}

Some researchers have proposed methods for automatic detection of microaneurysms and hemorrhages, presenting different solutions to the problem. Hatanaka et al. [10] present a classifier of candidate regions for red lesions that performs calculations using the three channels of the HSV (Hue Saturation Value) color space. Esmaeili et al. [11] extract the candidate regions for red lesions using the curvelt transform. Mariño et al. [12] extract the candidate regions by combining different filters and then a region growing process is used to reject the regions whose size does not fit in the red lesion pattern. Shahin et al. [13] use operators of mathematical morphology to extract the different objects of the retina and a classifier of regions based on neural networks is subsequently applied. Balasubramanian et al. [14] extract the candidate regions for red lesions with a method called Automatic Seed Generation (ASG), based on the intensity of the pixels and its similarity with the neighbors. Badea et al. [15] detect all objects of the retina with a proposed method, called Expanding Gradient Method (EGM), and then the candidate regions for red lesions are extracted. Kande et al. [16] use operators of mathematical morphology to extract candidate regions, proposing a classifier based on Support Vector Machines (SVM). Jaafar et al. [5], Niemeijer et al. [9] and Ravishankar et al. [17] also used techniques of mathematical morphology for detection of red lesions, getting satisfactory results.

Automatic detection of microaneurysms and hemorrhages is not an easy task. Some red lesions are very near the blood vessels, making it difficult to distinguish between them. The larger size hemorrhages have the same color of the vessels, differing only in geometry, but smaller microaneurysms and hemorrhages have color, texture and geometry very similar to the structure of blood vessels [18]. Furthermore, microaneurysms usually have a diameter less than $125 \mu \mathrm{m}$, and this small size and intensity variations in the background make the detection of microaneurysms a complicated task [8]. As noted in [2], fundus images often suffer from nonuniform illumination, poor contrast and noise, thus these images have to go through preprocessing phases. According to [16], the biggest challenges for detection of red lesions are the segmentation of microaneurysms in areas of low contrast and the presence of white lesions in the retina (exudates). It may also be mentioned that microaneurysms and hemorrhages have various sizes and shapes, making it difficult to perform detection of all of them in the image, mainly because the normal structures of the retina, as the fovea and the blood vessels, can be confused with lesions [19].

Several works use private images, such as $[9,10,12]$, or use images from undisclosed source, such as $[14,15]$. These facts impede the replication of the experiments of these works. The use of 
public databases of fundus images is important in order to prove the accuracy and reliability of each proposed method. Furthermore, some methods have a high runtime, such as [9, 17], taking many minutes to analyze each fundus images. This fact may preclude the application of these methods in processing of a big amount of fundus images.

In addition, many works focus on achieving high levels of sensitivity without prioritizing the specificity of the results. This fact makes these methods sensitive to many structures in the image, getting a high number of false positives. The sensitivity and specificity measures will be explained in details in Section 4.

In this paper, we propose a method which uses a public database of fundus images, achieving a good balance between the measures of sensitivity and specificity. The main contribution of the proposed method is the detection of blood vessels, where it was used a solution based on mathematical morphology, as will be explained in the following section.

\section{Materials AND Methods}

The implementation of the detection method proposed in this paper was performed in MATLAB (version 7). The accuracy of the method was tested in the public database of fundus images DIARETDB1 [20]. This database has a great variety of lesions that characterize the DR, besides being one of the most used in current publications. The DIARETDB1 was organized by a group of Finnish researchers, motivated by the fact that there is no common database in the publications of DR detection methods, making it impossible to prove the reliability and accuracy of each algorithm. The DIARETDB1 has a total of 89 fundus images (RGB) of size $1500 \times 1152$. Of this total, 84 images have characteristic signs of DR, such as microaneurysms, hemorrhages and exudates, and 5 images are of normal retinas.

In designing of the DIARETDB1 the regions with lesions were highlighted manually by four specialists in ophthalmology, which made this analysis individually in each fundus image. At the end, there were differences in the results of each of the four analyzes - not all lesions were unanimous decision, for example. So, it was considered the union of the four analyzes, forming the ground-truth of each image, which is also available in the DIARETDB1. However, one problem presented in this ground-truth is the low precision in the marking of lesions, since ophthalmologists delimited areas with a set of lesions and not each lesion itself. Thus, we chose to use in this work the ground-truth of the DIARETDB1 improved by [21] which identifies each lesion individually, merges microaneurysms and hemorrhages into the same image, and disregards the lesions highlighted by only one or two specialists. In this new ground-truth, among the 89 images, 53 images were defined with lesions and 36 images were defined without lesions.

The method for automatic detection of microaneurysms and hemorrhages proposed in this paper is based on mathematical morphology and has five stages. At the first stage, a preprocessing of the fundus images is performed in order to improve the contrast of these images. At the second stage, an enhancement of the low intensity structures (blood vessels, fovea and red lesions) is performed. At the third stage, the blood vessels are detected by the sum of morphological openings using linear structuring elements with different angles. At the fourth stage, occurs the subtraction of the blood vessels from the image obtained at the second stage. At the fifth and last stage, the region of the fovea is removed. Each stage is explained in details in the following sections. 


\subsection{Preprocessing}

First, the DIARETDB1 images were resized to $640 \mathrm{x} 480$ pixels in order to reduce the processing time of the method. Later, it was extracted the green channel of the RGB color space, which has better contrast when compared to the other channels [5]. The grayscale image f1 of each fundus image $f$ was obtained according to Eq. (1).

$$
f 1=\text { GreenChanel }(f)
$$

After, a Contrast-Limited Adaptive Histogram Equalization (CLAHE) algorithm was applied [22]. This technique consists in applying histogram equalization for each region in the image, enhancing the local contrast of each region. The resulting image was obtained according to Eq. (2).

$$
f 2=C L A H E(f 1)
$$

The resulting images of this stage are shown in Fig. 2.

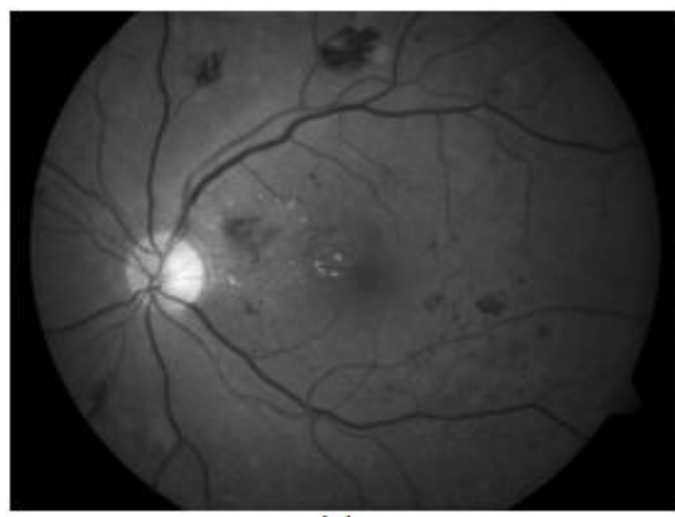

(a)

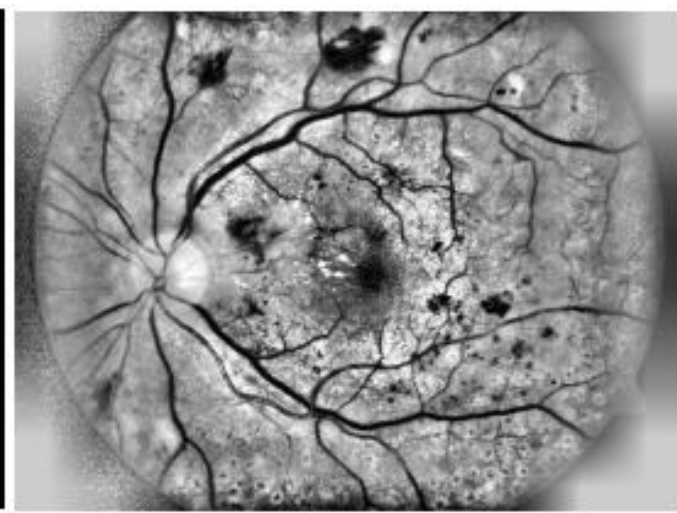

(b)

Figure 2. Preprocessing. (a) Image $f 1$ : green channel of the original fundus image. (b) Image $f 2$ : CLAHE.

\subsection{Enhancement of low intensity structures}

The purpose of this stage is an enhancement of the low intensity structures of the fundus images, and then use them as candidates for red lesions.

The first step of this stage is the application of the Alternating Sequential Filtering (ASF) in order to estimate the background of preprocessed images, according to Eq. (3). This filter is based on successive applications of morphological openings $\gamma$ and closings $\varnothing$, doubling the size of the structuring element $B$ at each iteration.

$$
f 3=\phi^{(n B)}\left(\ldots\left(\gamma^{2 E}\left(\phi^{(B)}\left(\gamma^{(B)}(f 2)\right)\right)\right) \ldots\right)
$$

The ASF operation was performed with 3 iterations, using a disk-shaped structuring element $B$ with radius of 5 pixels. After that, the resulting image of the CLAHE was subtracted from the resulting image of the ASF, obtaining an intensity inverted image, where the low intensity structures were enhanced in white (blood vessels, fovea and red lesions). Consequently, the high 
intensity structures (optical disc and exudates) were eliminated or were in a color close to black. The result of this operation is assigned to the image f4, according to Eq. (4).

$$
f 4=f 3-f 2
$$

Afterwards, regions of low contrast of $\mathrm{f} 4$, which could be associated with the optical disc or exudates, were eliminated using the H-minima transform, according to Eq. (5). This operator removes connected basins with contrast less than a threshold $h$, using for this purpose a morphological reconstruction based on erosion, denoted by $\mathrm{R}^{*}$.

$$
f 5=\operatorname{Hmin}_{h}(f 4)=R_{f 4}^{*}(f 4+h)
$$

In the next step, the image f5 was binarized using the morphological operator of regional minimum RMIN, according to Eq. (6). This operator converts a grayscale image to binary format without using any threshold, and is also based on morphological reconstruction by erosion. In addition, after the execution of the RMIN operator, the pixel values of $\mathrm{f} 6$ were inverted.

$$
f 6=R M I N(f 5)=R_{f 5}^{*}(f 5+1)-f 5
$$

The resulting images of this stage are shown in Fig. 3.

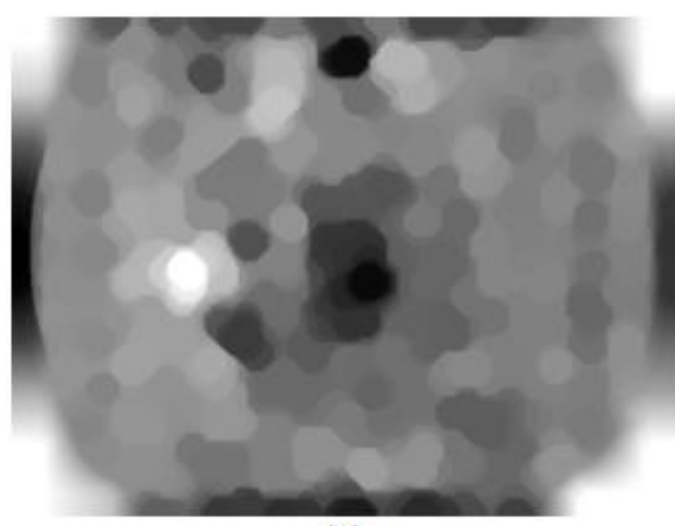

(a)

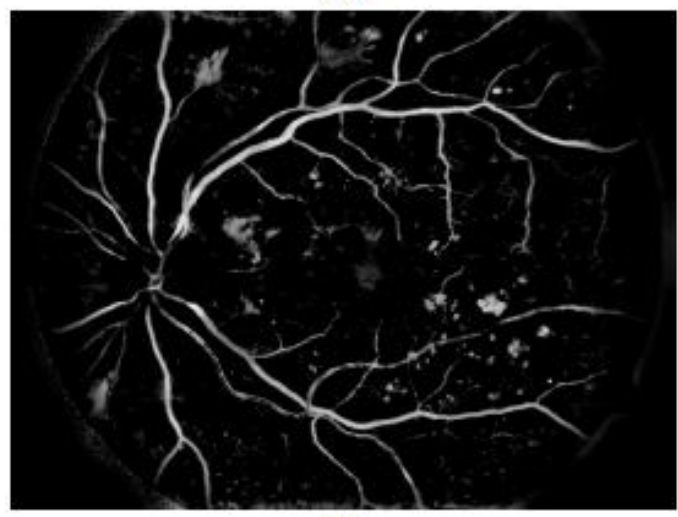

(c)

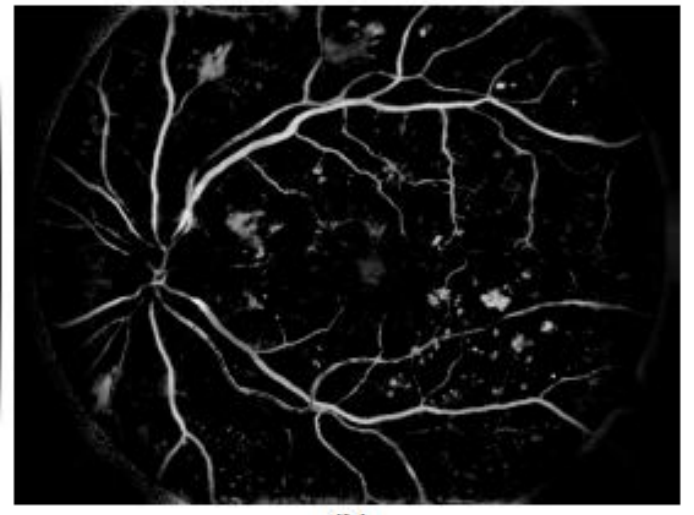

(b)

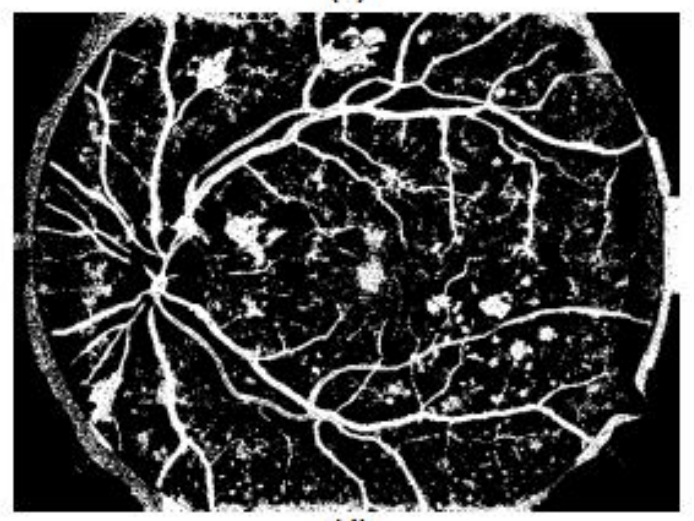

(d)

Figure 3. Enhancement of low intensity structures. (a) Image $f 3$ : ASF. (b) Image $f 4$ : low intensity structures enhanced in white. (c) Image $f 5$ : H-minima transform. (d) Image f6: regional minimum with inverted pixel values. 


\subsection{Detection of blood vessels}

For the detection of the blood vessels, the ASF was again performed on the resulting image of the CLAHE, using now only one iteration. It was used a disc-shaped structuring element $B$ with radius of 3 pixels, according to Eq. (7).

$$
f 7=\phi^{(n D)}\left(\ldots\left(\gamma^{2 B}\left(\phi^{(D)}\left(\gamma^{(B)}(f 2)\right)\right)\right) \ldots\right)
$$

Following the same idea of the enhancement of the low intensity structures, we obtained the image f8, without variations in the background, subtracting the resulting image of the CLAHE from image f7, according to Eq. (8).

$$
f 8=f 7-f^{2}
$$

Then, the detection of the blood vessels was implemented using the morphological opening of f8 with multi-scale structuring elements through a similar process to that proposed in [9]. The morphological opening $\gamma$ was performed 12 times on the image f8, using linear structuring elements with 12 different angles, ranging 15 degrees to each structuring element (namely $0{ }^{\circ}, 15$ ${ }^{\circ}, 30^{\circ}$, and so on up to $165^{\circ}$ ). As the blood vessels have a linear and elongated geometric feature, the length of the structuring element was defined with the value 40 pixels, so that the openings do not consider rounded structures that could be associated with the fovea or red lesions. Finally, the 12 images obtained were added, getting the image f9, containing a sketch of the blood vessels, according to Eq. (9), where $i$ identifies each of the openings and $n$ is defined with the value 12 .

$$
f^{9}=\sum_{i=1}^{i=n} \gamma^{(B i)} f 8+\gamma^{(B i+1)} f 8+\cdots+\gamma^{(B n)} f^{8}
$$

In the next step, the detection of the blood vessels was performed from a morphological reconstruction by dilation, denoted by $R$, according Eq. (10). This operation reconstructs the connected components of the image f8 which also exist in the image f9, which contains the sketch of the vessels. In this operation, we used a diamond-shaped structuring element with radius of 1 pixel.

$$
f 10=R_{f o}(f 9)
$$

Finally, the image f10, that contains the detected blood vessels, was binarized using the morphological operator of regional minimum RMIN, according to Eq. (11).

$$
f 11=R M I N(f 10)^{c}
$$

The resulting images of this stage are shown in Fig. 4(a)-(e).

\subsection{Elimination of blood vessels}

This stage consists of removing the blood vessels obtained in the previous stage from image f6, which contains the low intensity structures. From this subtraction, the image f12 was obtained, containing the candidate regions for red lesions, according Eq. (12).

$$
f 12=f 6-f 11
$$

The resulting image of this stage is shown in Fig. 4(f). 


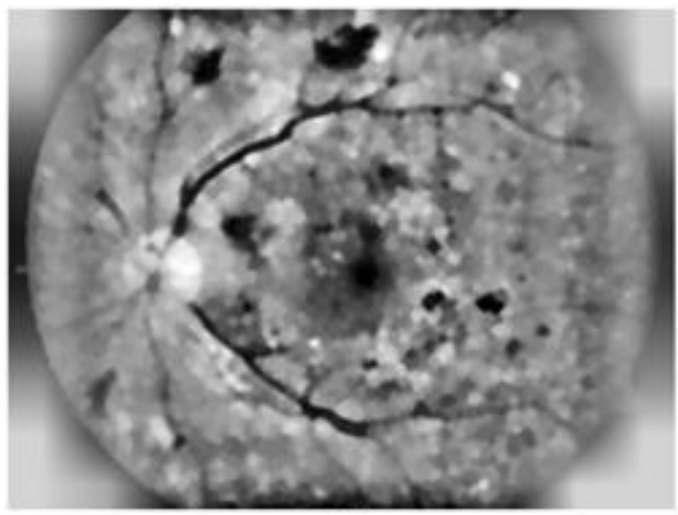

(a)

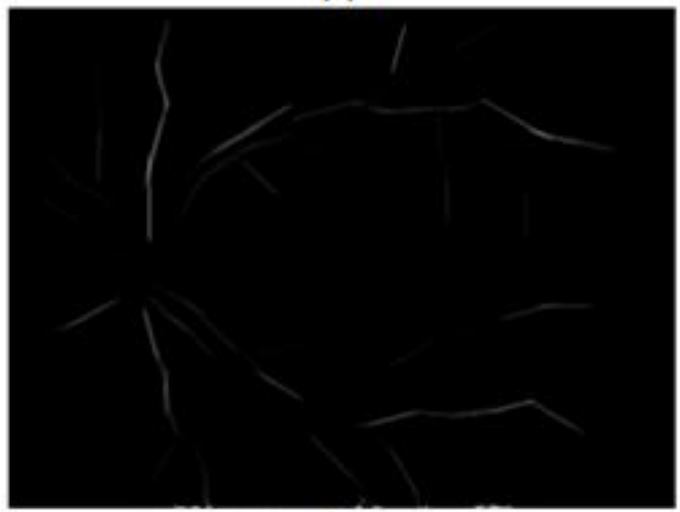

(c)

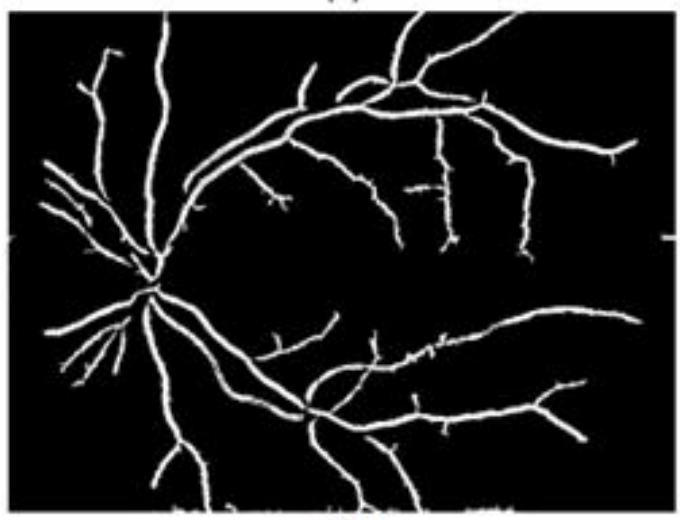

(e)

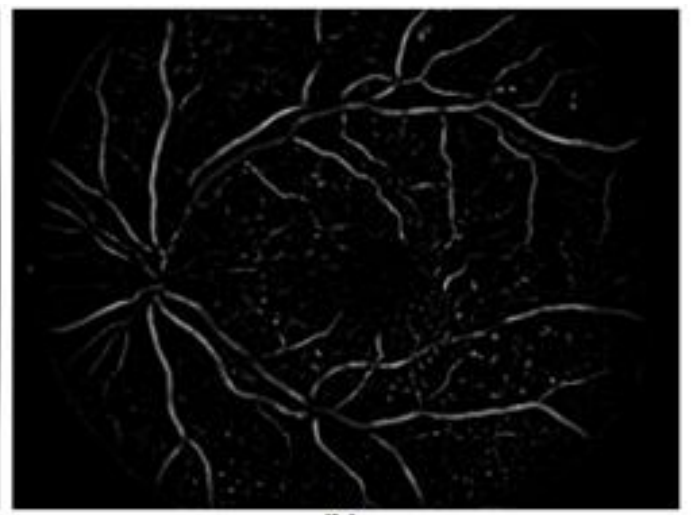

(b)

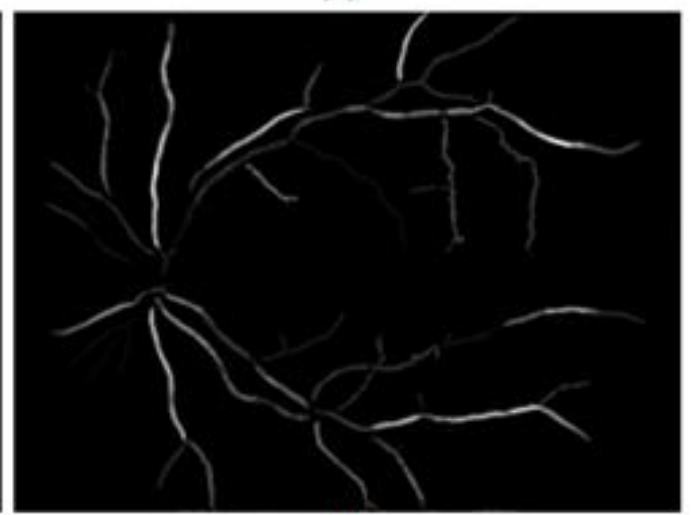

(d)

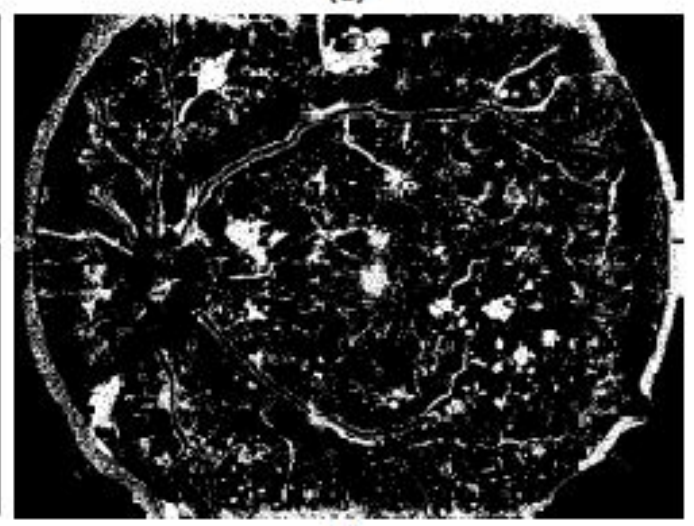

(f)

Figure 4. Detection and elimination of blood vessels. (a) Image $f 7$ : ASF. (b) Image $f 8$ : reduction of variations in the background. (c) Image f9: sum of morphological openings. (d) Image f10: morphological reconstruction of the blood vessels. (e) Image $f 11$ : regional minimum with inverted pixel values. (f) Image $f 12$ : elimination of the blood vessels.

\subsection{Elimination of the fovea}

This is the last stage of the proposed method. In order to improve the detection accuracy, we removed the fovea region from image 112 , obtained in the previous stage. First, it was applied a method that identifies the center of the fovea, based on characteristics of the anatomy of the retina, which considers the spatial relationship between the diameter of the optical disc and the region of the fovea [23]. It was obtained here a binary image called FoveaCenter, which has only 
a pixel with value 1 , which identifies the center of the fovea. After that, it was performed a morphological dilation with a disc-shaped structuring element, with a radius of 25 pixels, since the fovea has circular characteristics and has a similar size to this value. The result of this operation is assigned to the image f13, according to Eq. (13).

$$
f 13=\delta^{(B)} \text { (FoveaCenter) }
$$

Later, the fovea was removed by subtracting the image f13 from image f12 (which contains the candidates for red lesions), according to Eq. (14).

$$
f 14=f 12-f 13
$$

Afterwards, some morphological operations were performed on the image f14 in order to refine the final image. Firstly, it was performed a morphological operation named FILL, which fills small gaps in the image. After, it was performed an opening morphological with a diamondshaped structuring element, with a radius of 1 pixel, in order to remove some noises in the image. Next, it was performed a morphological dilation, with the same structuring element, in order to slightly enlarge the remaining structures. From these operations, it was obtained the image f15, according to Eq. (15).

$$
f 15=\delta^{(B)}\left(\gamma^{(E)}(F I L L(f 14))\right)
$$

Finally, it was applied a mask provided by the DIARETDB1, which removes the areas outside the circumference of the retina, according to Eq. (16), thereby obtaining the final image of the method, f16, containing the microaneurysms and hemorrhages.

$$
f 16_{(x y)}= \begin{cases}0, & \text { if } \operatorname{mask}_{(x, y)}=0 \\ f 15_{(x y)}, & \text { otherwise }\end{cases}
$$

The resulting images of this stage are shown in Fig. 5. The flowchart in Fig. 6 summarizes the steps of the detection method, showing the resulting image of each step, from the original image, f1, until the final image f16, containing the microaneurysms and hemorrhages. 


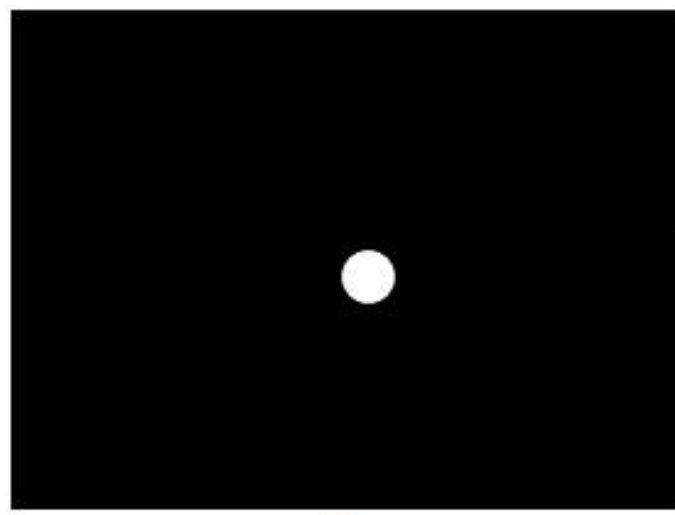

(a)

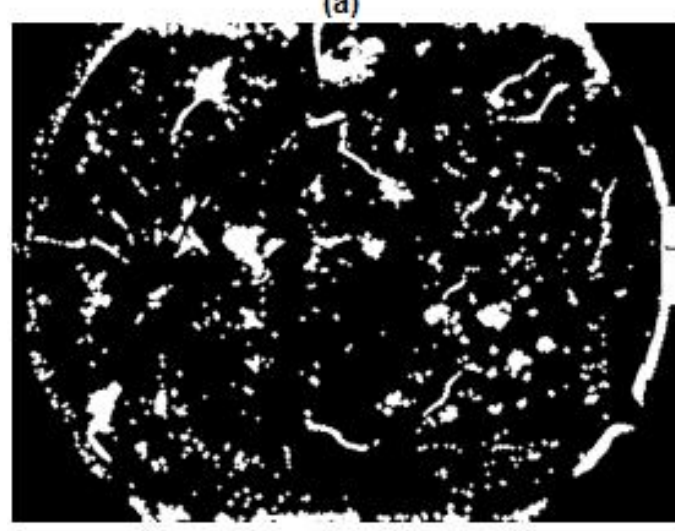

(c)

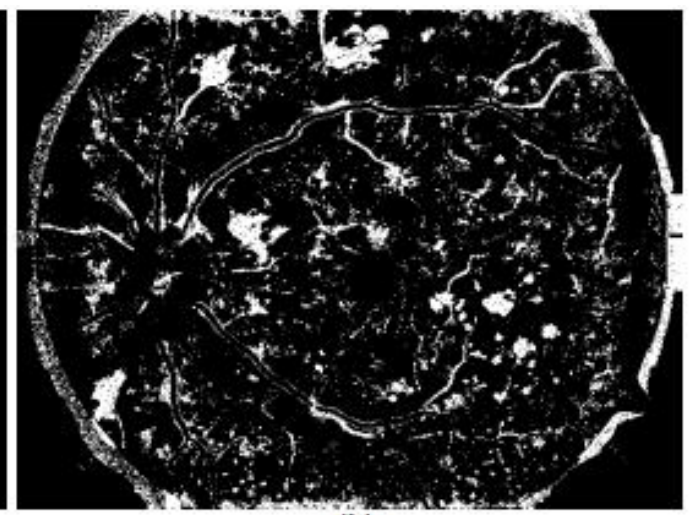

(b)

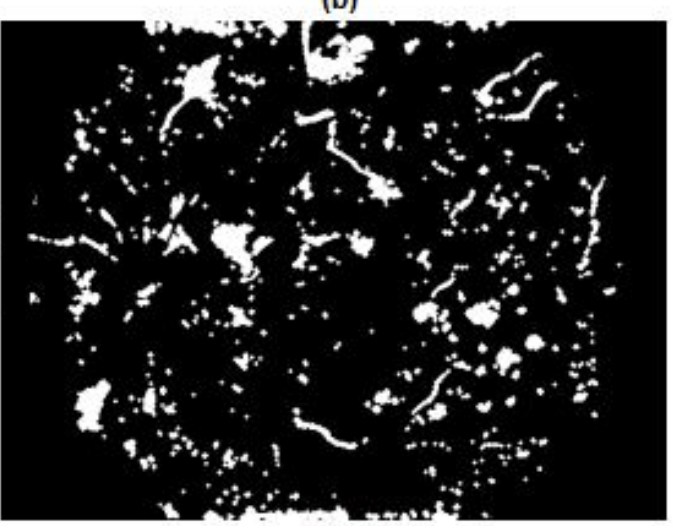

(d)

Figure 5. Elimination of the fovea. (a) Image $f 13$ : dilation of the center of the fovea. (b) Image $f 14$ : elimination of the region of the fovea. (c) Image f15: FILL, opening and dilation. (d) Image $f 16$ : mask.

\section{RESULTS AND DISCUSSIONS}

The sensitivity and specificity measures were used to quantify the results achieved by the detection method. These measures were chosen based on the related work, where most publications use these measures. For a clear understanding of these measures, it is necessary to conceptualize some classifications used in laboratory tests for detection of diseases. When a test result is positive, the individual can manifest the disease, which is called "True Positive" (TP) or cannot express it, which is known as "False Positive" (FP). On the other hand, when the result is negative, the individual cannot have the disease, which is known as "True Negative" (TN) or can have it, what is called "False Negative" (FN) [24].

The sensitivity is defined as the ability of a test to detect correctly people with a disease or condition. [25] The sensitivity is calculated according to Eq. (16).

$$
\text { Sensitivity }=\frac{T P}{T P+F N}
$$

The specificity is defined as the ability of a test to exclude properly people without a disease or condition [25]. The specificity is calculated according to Eq. (17).

$$
\text { Specificity }=\frac{T N}{T N+F P}
$$


It is important to have some balance between the values of sensitivity and specificity. Get high values for sensitivity and low values for specificity means that the method detected most lesions (true positives), but other structures were erroneously classified as lesions (false positives). On the other hand, get high values for specificity and low values for sensitivity means that the method deleted correctly regions without lesions (true negative) but was not able to identify all lesions (true positives).

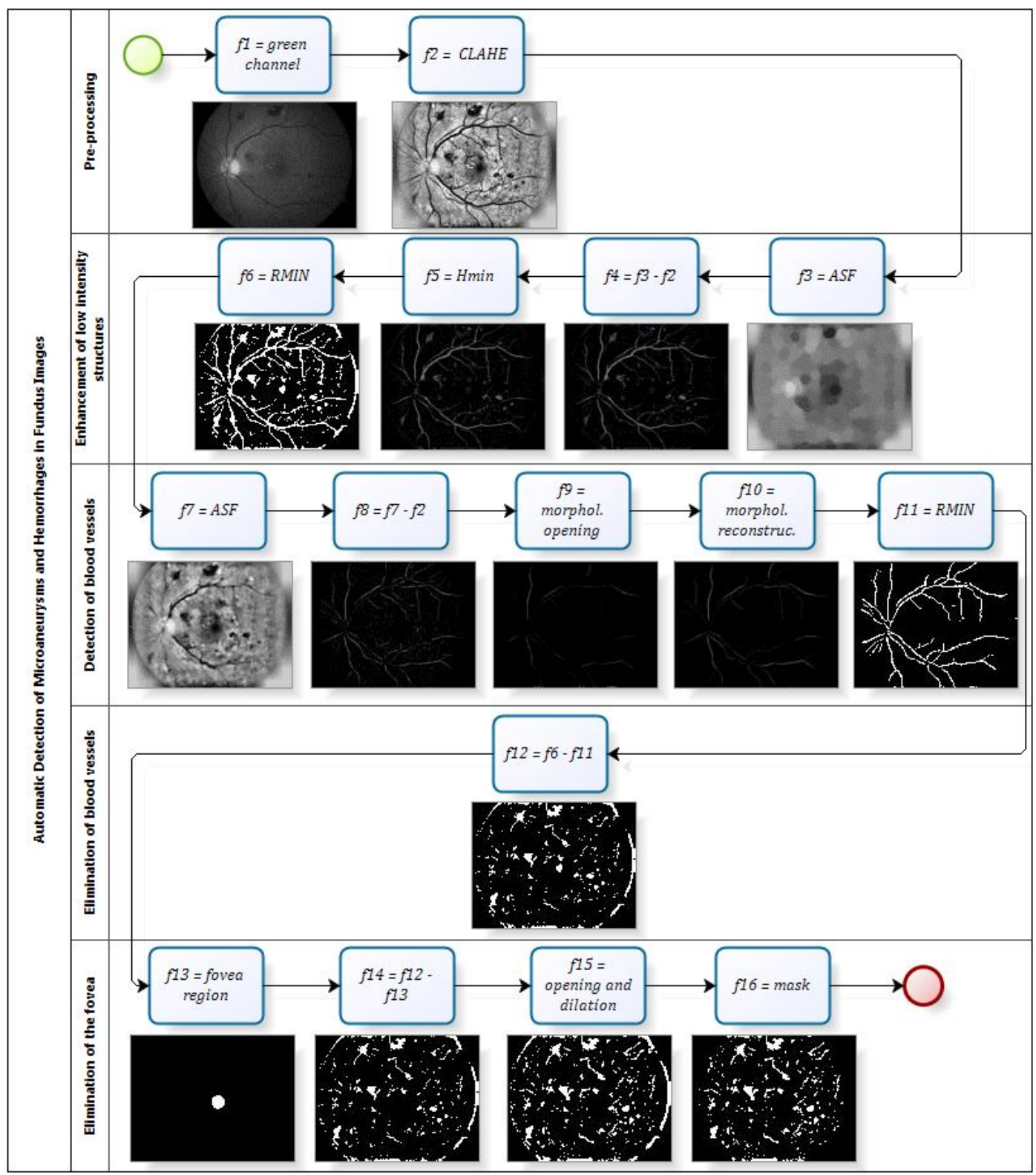

Figure 6. Flowchart of the method for detection of microaneurysms and hemorrhages

The performance of the method was tested on images with incidence of lesions provided by the DIARETDB1. The accounting of the pixels in TP, FN, FP and TN was performed for each resulting image of the method. Table 1 shows the average values of TP, FN, FP and TN for each fundus image. 
International Journal of Computer Science \& Information Technology (IJCSIT) Vol 5, No 5, October 2013

Table 1. Average values of TP, FN, FP e TN

\begin{tabular}{|c|c|c|c|}
\hline True Positives & False Negatives & False Positives & True Negatives \\
\hline 1809.62 & 329.73 & 23012.45 & 282048.18 \\
\hline
\end{tabular}

Fig. 7(a) shows the green channel of a fundus image, containing microaneurysms and hemorrhages. Fig. 7(b) shows the ground-truth of the image. Fig. 7(c) shows the detected microaneurysms and hemorrhages. Figs. 7(d)-(g) show the pixels of true positives, false negatives, false positives and true negatives, respectively. 


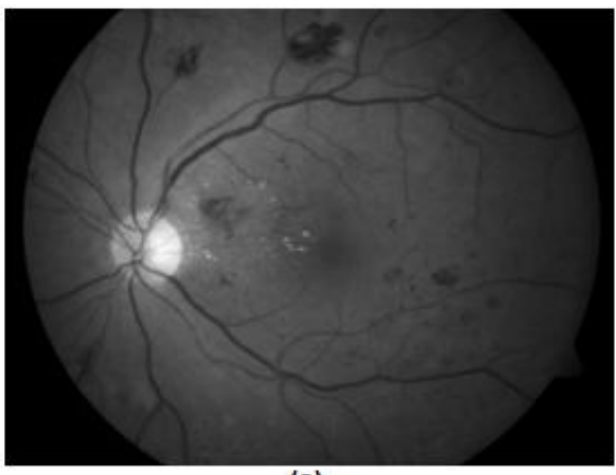

(a)

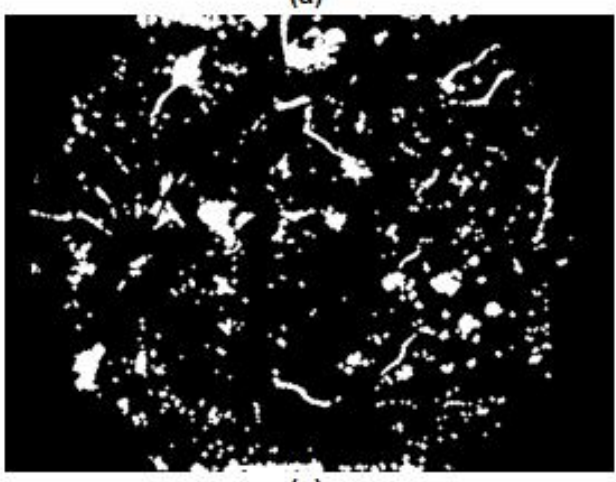

(c)

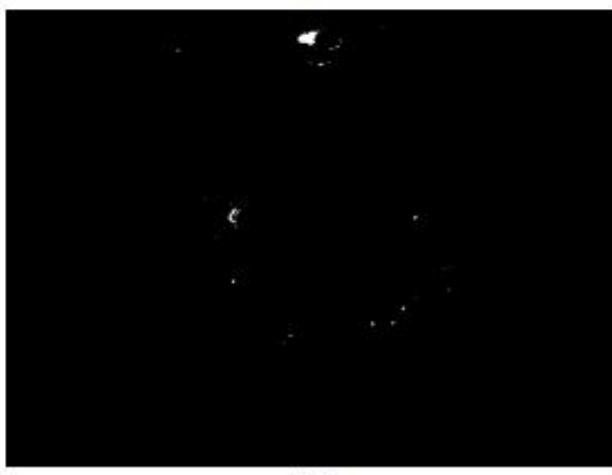

(e)

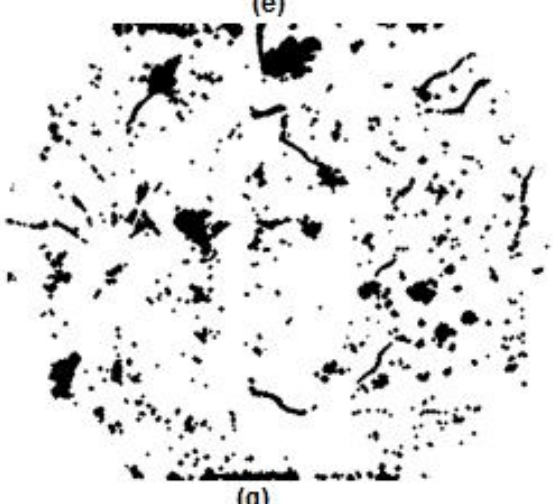

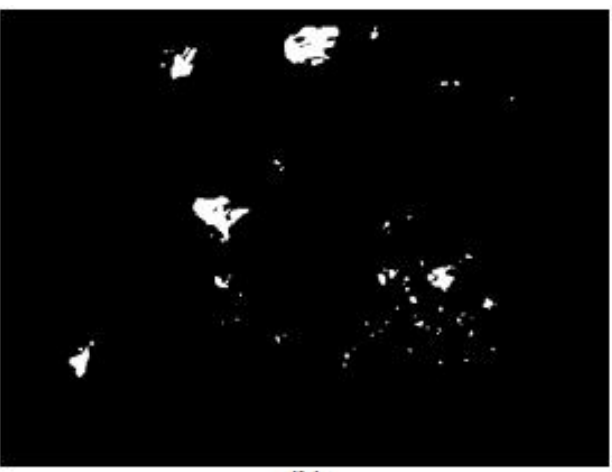

(b)

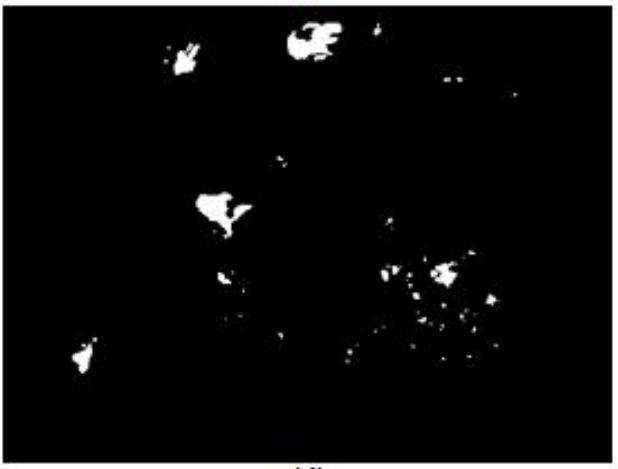

(d)

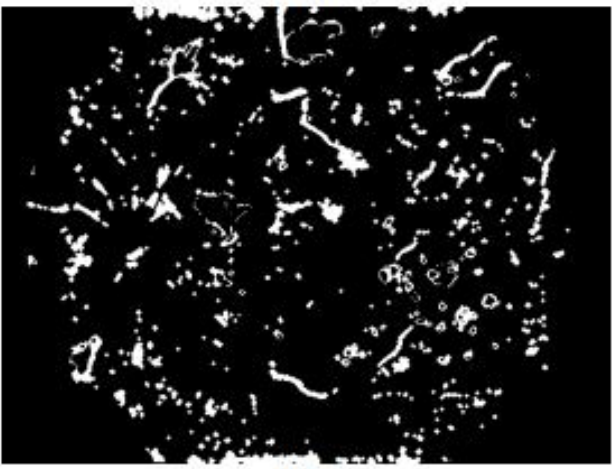

(f)

Figure 7. Illustration of false positives/negatives and true positives/negatives used to calculate the sensitivity and specificity measures. (a) Green channel of the original fundus image. (b) Ground-truth image. (c) Microaneurysms and hemorrhages detected. (d) True positive pixels. (e) False negative pixels. (f) False positive pixels. (g) True negative pixels. 
Each resulting image of the method was compared with its ground-truth, where it was performed an analysis based on the area of each region. The sensitivity and specificity measures were calculated by image, and after it was performed the general average of the values obtained for each image, where the method achieved a sensitivity of $87.69 \%$ and a specificity of $92.44 \%$. Table 2 compares the results obtained by the method with other works of literature.

Table 2. Comparison of methods for detection of microaneurysms and hemorrhages

\begin{tabular}{|c|c|c|c|}
\hline Author & $\begin{array}{c}\text { Publicly available } \\
\text { images }\end{array}$ & Sensitivity & Specificity \\
\hline Jaafar et al. [5] & $\begin{array}{l}\text { Yes: DIARETDB0 } \\
\text { and DIARETDB1 }\end{array}$ & $98.8 \%$ & $86.2 \%$ \\
\hline Niemeijer et al. [9] & No & $100 \%$ & $87 \%$ \\
\hline Ravishankar et al. [17] & $\begin{array}{l}\text { Some: DIARETDB0, } \\
\text { DRIVE and STARE }\end{array}$ & $95.1 \%$ & $90.5 \%$ \\
\hline Balasubramanian et al.[14] & Not specified & $87 \%$ & $95.53 \%$ \\
\hline Badea et al. [15] & Not specified & Not specified & Not specified \\
\hline Kande et al. [16] & $\begin{array}{l}\text { Yes: STARE, } \\
\text { DIARETDB } 0 \text { and } \\
\text { DIARETDB } 1\end{array}$ & $100 \%$ & $91 \%$ \\
\hline Shahin et al. [13] & $\begin{array}{c}\text { Yes: STARE, } \\
\text { DRIVE, } \\
\text { DIARETDB0 and } \\
\text { DIARETDB } 1\end{array}$ & $88 \%$ & $100 \%$ \\
\hline Roychowdhury et al. [26] & Yes: DIARETDB1 & $75.5 \%$ & $93.73 \%$ \\
\hline Garcia et al. [27] & No & $100 \%$ & $60 \%$ \\
\hline Hatanaka et al. [10] & No & $80 \%$ & $80 \%$ \\
\hline Esmaeili et al. [11] & Yes: DIARETDB1 & $94 \%$ & $87 \%$ \\
\hline Mariño et al. [12] & No & $78.5 \%$ & Not specified \\
\hline Proposed method & Yes: DIARETDB1 & $87.69 \%$ & $92.44 \%$ \\
\hline
\end{tabular}

The proposed method achieved satisfactory results, but there are some problems that should be mentioned. Fig. 8 illustrates the effect of false positives together with the red lesions that were detected. These structures are mainly caused by residues of blood vessels, where there was inefficiency of the method to eliminate some vessels without removing lesions close to them. Another problem of the method, that decreased the sensitivity, occurred in the fifth stage, where the elimination of the region of the fovea removed some lesions overlapping to this region. Another factor that affected the sensitivity was not identifying some microaneurysms occupying an area very small. Due to its very small size, some of these lesions were confused with noise and were improperly removed. It may also be mentioned that the non-uniform illumination of some images generated false positive results, which decreased the specificity of the method. 


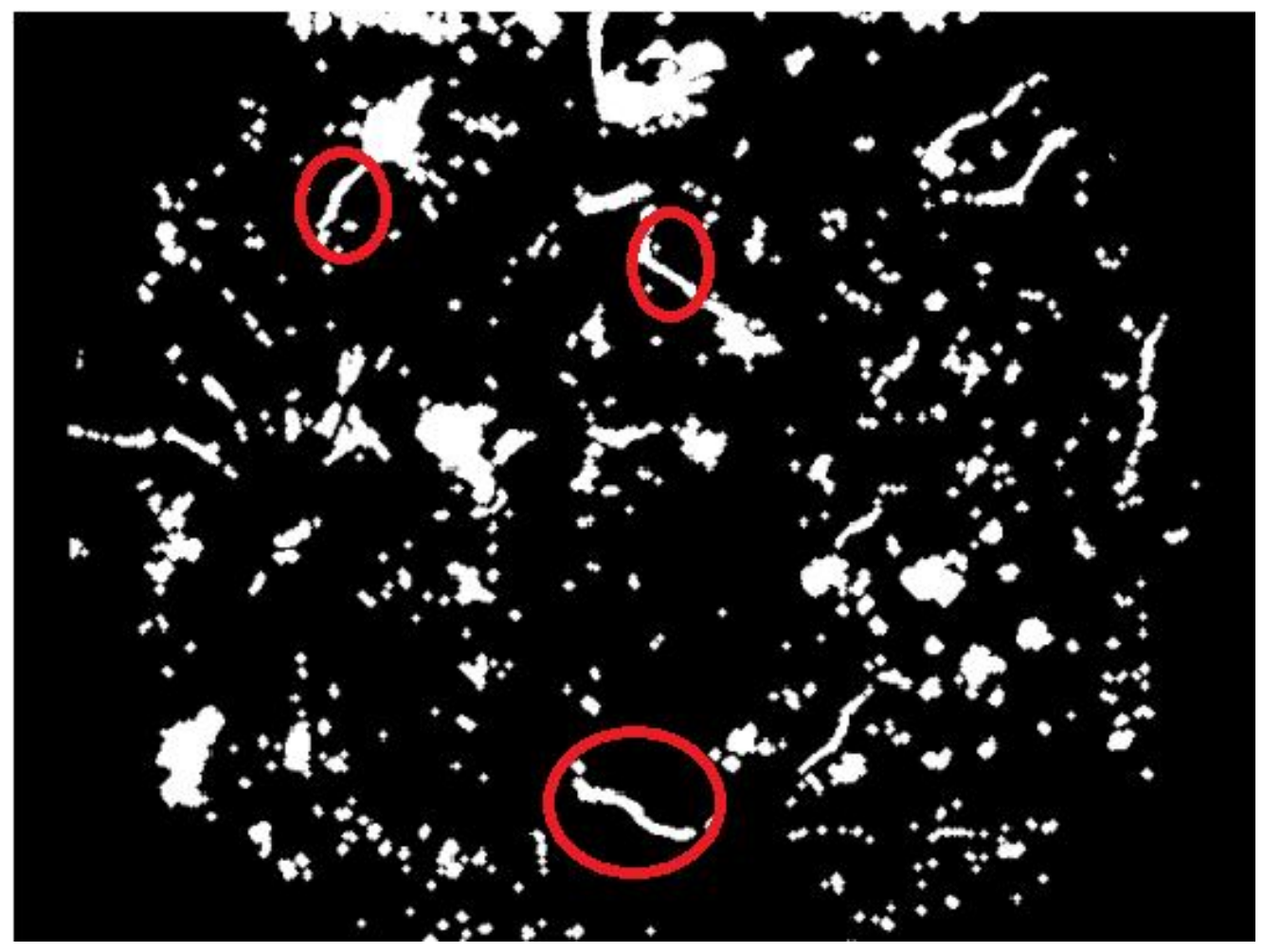

Figure 8 . False positives caused by residues of blood vessels

On the other hand, the method achieved interesting results in the third stage (detection of blood vessels), getting good precision with the solution presented, based on the sum of morphology openings and reconstruction by dilation, which can be considered the major contribution of this paper. The enhancement of low intensity structures also was successful, since areas of the optical disc and white lesions did not affect the results significantly.

The method also obtained attractive results regarding its runtime, because its low complexity. Each image was analyzed with about 5 seconds of processing, when the method was tested on a machine with Intel Core 2 Duo $(2.20 \mathrm{GHz})$ processor with $3 \mathrm{~GB}$ of RAM. This fact may be a differential of the method for purposes of large scale processing of fundus images.

\section{Conclusions And Future Trends}

This paper presented a novel method for automated detection of red lesions in fundus images. The appearance of these lesions in the retina is characterized as the earliest sign of diabetic retinopathy, a complication arising from diabetes, which affects more and more people in the world causing irreparable visual impairments. From the alarming predictions of the World Health Organization about the significant growth of diabetes cases in the coming years, become necessary measures by governments for mass screening of diabetic populations. These actions would allow the screening of diabetic patients periodically, avoiding the evolution of the diabetic retinopathy. In this context, a system for automated detection of microaneurysms and hemorrhages would be extremely useful, being able to identify these lesions more quickly than manual inspections performed by ophthalmologists. Moreover, such systems would afford the processing of a large amount of images, using for this purpose retinal images captured in a noninvasive manner. 
The proposed method achieved sensitivity and specificity of $87.69 \%$ and $92.44 \%$, respectively. Therefore, it can be concluded that the method was successful in detecting the microaneurysms and hemorrhages, achieving performance comparable to other literature methods.

As future work, it will be developed methods to detect other lesions related to diabetic retinopathy and other diseases that affect the retina.

\section{REFERENCES}

[1] World Health Organization, International Diabetes Federation, "Diabetes", http://www.who.int/mediacentre/factsheets/fs312/en/index.html (Jun. 5, 2013).

[2] T. Walter, P. Massin, A. Erginary, R. Ordonez, C. Jeulin, and J. Klein, (2007) "Automatic detection of microaneurysms in color fundus images,” Medical Image Analysis, Vol. 11, pp. 555-566.

[3] B. Sujithkumar, and V. Singh, (2012) "Automatic Detection of Diabetic Retinopathy in Non-dilated RGB Retinal Fundus Images", International Journal of Computer Applications, Vol. 47, No. 19, pp. 26-32.

[4] S. C. Lee, E.T. Lee, R. M. Kingsley, Y. Wang, D. Russell, R. Klein, and A. Warn, (2001) "Comparison of diagnosis of early retinal lesions of diabetic retinopathy between a computer system and human experts", Arch. Ophthalmol, Vol. 119, pp. 509-515.

[5] H. F. Jaafar, A. K. Nandi, and W. Al-Nuaimy, (2011) "Automated detection of red lesions from digital colour fundus photographs", Proceedings: Conf Proc IEEE Eng Med Biol Soc, pp. 6232-6235.

[6] L. Albuquerque, "O que é a retinopatia diabética?", http://www.institutoprovisao.com.br/diabetes.html/ (Jun. 5, 2013).

[7] T. A. Ciulla, A. G. Amador, and B. Zinman, (2003) "Diabetic Retinopathy and Diabetic Macular Edema: Pathophysiology, screening, and novel therapies", Diabetes Care, Vol. 26, No. 9, pp. 26532664.

[8] D. Saleh, and C. Eswaran, (2012) "An automated decision-support system for non-proliferative diabetic retinopathy disease based on MAs and HAs detection", Computer Methods and Programs in Biomedicine, Vol. 11, No. 1, pp. 186-196.

[9] M. Niemeijer, B. V. Ginneken, J. Staal, M. S. Suttorp-Schulton and M. D. Abramoff, (2005) "Automatic detection of red lesions in digital color fundus photograph", IEEE Trans Med Imaging, Vol. 24, No. 5, pp. 584-592.

[10] Y. Hatanaka, T. Nakagawa, Y. Hayashi, T. Hara, and H. Fujita, (2008) "Improvement of Automated Detection Method of Hemorrhages in Fundus Images", Proceedings: 30th Annual International IEEE EMBS Conference, pp. $5429-5432$.

[11] M. Esmaeili, H. Rabbani, and A. Dehnavi, (2010) "A new curvelet transform based method for extraction of red lesions in digital color retinal images", Proceedings: "IEEE International Conference On Image Processing", pp. 4093-4096.

[12] C.Mariño, E. Ares, M.G.Penedo, M. Ortega, N. Barreira, and F. Gomez-Ulla, "Automated three stage red lesions detection in digital color fundus images", Wseas Transactions on Computers, Stevens Point, v. 7, n. 4, 2008, pp. 207-215.

[13] E. M. Shahin, T. E.Taha, W. Al-Nuaimy, S. El Rabaie, O. F. Zahran, and F. E. Abd El-Samie, (2012) "Automated Detection of Diabetic Retinopathy in Blurred Digital Fundus Images", Proceedings: Computer Engineering Conference (ICENCO), pp. 20-25.

[14] S. Balasubramanian, S. Pradhan, and V. Chandrasekaran, (2008) "Red Lesions Detection in Digital Fundus Images", Proceedings: IEEE International Conference On Image Processing, pp. 2932 - 2935.

[15] P. Badea, D. Danciu, and L. Davidescu, (2008) "Preliminary results on using an extension of gradient method for detection of red lesions on eye fundus photographs", Proceedings: IEEE International Conference On Automation, Quality And Testing, Robotics, pp. 43-48.

[16] G. B. Kande, T. S. Savithri, and P. V. Subbaiah, (2010) "Automatic Detection of Microaneurysms and Hemorrhages in Digital Fundus Images”, Journal Of Digital Imaging, Vol. 23, No. 4, pp. 430-437.

[17] S. Ravishankar, A. Jain, and A. Mittal, (2009) "Automated feature extraction for early detection of diabetic retinopathy in fundus images", Proceedings: IEEE Conference On Computer Vision And Pattern Recognition, pp. 210-217.

[18] N. Silberman, K. Ahlrich, R. Fergus, and L. Subramanian, (2010) "Case for Automated Detection of Diabetic Retinopathy", Aaai Spring Symposium Series.

[19] M. D. Abràmoff, M. K. Garvin, and M. Sonka, (2010) "Retinal Imaging and Image Analysis", IEEE Reviews In Biomedical Engineering, Vol. 3, pp. 169-208.

[20] T. Kauppi, V. Kalesnykiene, J. K. Kamarainen, L. Lensu, I. Sorri, A. Raninen, R. Voutilainen , H. Uusitalo, H. Kalviainen, and J. Pietila, (2007), "DIARETDB1: diabetic retinopathy database and evaluation protocol," Technical report, Faculty of Medicine, University of Kuopio, Finland.

[21] L. Bagesteiro, "Detecção de Lesões Vermelhas em Imagens de Fundo do Olho", Universidade Federal do Pampa, Campus Alegrete, Curso de Ciência da Computação, 2013.

[22] K. Zuiderveld, (1994 ) "Contrast Limited Adaptive Histogram Equalization," Chapter VIII.5, Graphics Gems IV. P.S. Heckbert (Eds.), Cambridge, MA, Academic Press, pp. 474-485.

[23] D. Welfer, J. Sharcanski, and D. R. Marinho, (2010) "Fovea center detection based on the retina anatomy and mathematical morphology", Computer Methods and Programs in Biomedicine, Vol. 104, No. 3, pp. 397-409. 
International Journal of Computer Science \& Information Technology (IJCSIT) Vol 5, No 5, October 2013

[24] OBID - Observatório Brasileiro De Informações Sobre Drogas, "Sensibilidade e Especificidade", http://www.obid.senad.gov.br/portais/OBID/conteudo/index.php?id_conteudo=11450\&rastro=PESQ UISAS+E+ESTAT\%C3\%8DSTICAS\%2FConceitos+Estat\%C3\%ADsticos/Sensibilidade+e+Especifi cidade (Jun. 5, 2013).

[25] PTB - $\quad$ Programa Telessaúde $\quad$ Brasil, "Abordagem Diagnóstica", http://www.telessaudebrasil.org.br/apps/calculadoras/?page=1 (Jun. 5, 2013).

[26] S. Roychowdhury1, D. D. Koozekanani, and K. K. Parhi, (2012) "Screening Fundus Images for Diabetic Retinopathy”, Proceedings: Conference Record of the Forty Sixth Asilomar, pp. 1641-1645.

[27] M. Garcia, C. Sanchez, M. I. Lopez, A. Diez, and R. Hornero, (2008) "Automatic Detection of Red Lesions in Retinal Images Using a Multilayer Perceptron Neural Network", Proceedings: International Conference of IEEE Engineering in Medicine and Biology Society, pp. 5425-5428.

\section{Authors}

Sérgio Bortolin Júnior is a Master's student in electrical engineering at the Federal University of Pampa (UNIPAMPA), Brazil, where also works as analyst of information technology. His main research interests are image processing and web programming. Email: sergiojunior@unipampa.edu.br

Daniel Welfer is a computer science Professor at the Federal University of Pampa - UNIPAMPA, campus Alegrete. His areas of interest are medical image processing and analysis, mathematical morphology, computer vision, DICOM formats, and hospital information systems. He recently became a permanent member in the Graduate Program in Electrical Engineering (PPGEE) at the Federal University of Pampa. Email: danielwelfer@unipampa.edu.br 